\title{
Research On Virtual Simulation Experiment Teaching Of Mechanical Design Based On Virtual Prototyping Technology
}

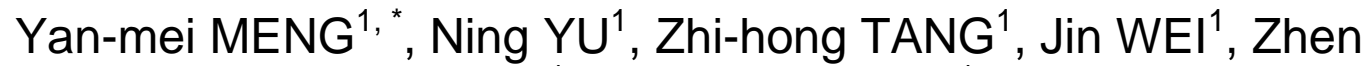 \\ $\mathrm{DONG}^{1}$ and Xian-wang $\mathrm{LI}^{1}$ \\ ${ }^{1}$ College of Mechanical Engineering, Guangxi University, Nanning 530004, China \\ *gxu_mengyun@163.com
}

Keywords: Virtual prototyping, Innovative design for machinery, Simulation experiment.

\begin{abstract}
This paper studies the teaching method of mechanical innovation design based on virtual prototyping technology. According to the specific requirements of basic course and specialized course on mechanical design, we pay attention to comprehensive training for the virtual modeling, motion simulation and optimization design. At the same time, combined with the practical aspects of student's extra-curricular, such as curriculum design, graduation project, experimental skills project, participation in teacher's research projects and discipline competitions, we organize the independent practice in the idea of progressive thinking and knowledge construction, to inspire students to autonomously study the corresponding tasks of product digital design in different stages, which can effectively solve the key problems that the undergraduates in mechanical majors are week in the practical ability of innovative design.
\end{abstract}

\section{Introduction}

In the 1980s, the concept of virtual experiment platform was put forward by Professor William Wolf (Willian Wolf) at the University of Virginia, the United States. It has been widely studied and applied in many foreign universities so far. The goal of the experimental platform which is a comprehensive application of virtual reality, multimedia, human-computer interaction, database and network communication technology is to build a realistic information and network environment including Operating environment Experimental operating environment and experiment object. And students can carry out efficient, safe and economical experiment in the open, independent and interactive virtual environment. Finally it can achieve the teaching effect that real experiments do not have or are difficult to achieve. The virtual simulation experiment teaching has obvious advantages especially involving in high risk or extreme environment, inaccessible or irreversible action, and the high cost, high consumption of large scale or comprehensive experimental projects $[1,2,3,4]$. On the other hand, through the virtual simulation experiment teaching, we can partially make up for the problems in the process of developing students' practical teaching, such as the number of students above normality, the shortage of experimental funds, the lack in practical depth and narrow range, and it can also significantly improve the efficiency of practical teaching funds and save funds of practical teaching. Virtual experiment platform provides an advanced and flexible experimental environment, which makes the classroom teaching no longer confined to the physical space, limited equipment, and it makes the teaching and learning, methods being invisible and extensive. It achieves the teaching purposes of interactive teaching, sharing resources, all-weather information experiment and non-constraints to time and space. It can be said that the 
virtual simulation experiment has a far-reaching and huge influence on traditional experimental teaching in ideology, system, modes, contents, methods and means, etc. It is an important part of higher education information technology, which reflects reform and innovation of experiment teaching, the integration of information technology and education development and the comprehensive integration of disciplines [5,6].

\section{The Setting Of Virtual Simulation Experiment Teaching Of Mechanical Design}

Virtual simulation experiment teaching of mechanical design includes virtual experiment of mechanical design basic and virtual experiment of mechanical product digital design. The former is composed by Mechanical Drawing, Computer Plots, Mechanical parts and principle, Machine Design and other basic course. The latter is the integration of Computer Aided Engineering (CAE) with Virtual Prototyping Technology (VPT) and other specialized courses. Teaching experiment is planned by three levels: basic type, comprehensive design, and innovative research.

The virtual simulation experiment of mechanical is designed through the studies of two-dimensional, three-dimensional software, familiar with the computer aided design (CAD)of typical parts, observing the components and the movement of typical parts in virtual environment (take the gear pump and the reduction box as an example), and the studies of parts mapping and virtual assembly. All of these not only make the students have a strong sense of perception, but also cultivate their ability to design and provide a virtual reality platform for the open creative design, which develops the students' creative awareness. Learning mechanical design method, they will obtain thinking methods in mechanical structure, mechanical design and innovative practice.

The digital design experiments of mechanical products emphasize that in a virtual reality environment, building a digital model can reflect the product feature with computer. it simulates motion and dynamic characteristics of the system in real environment, and then the virtual test that can't be done by physical prototypes is completed with the streamlining and optimized system relying on simulation results. Finally the product-level optimized design scheme can be obtained without physical prototype. Students can build the models of products with CAD software, then simulate and analyze them with engineering analysis software, such as ANSYS, ADMAS, and so on, and finally display the results of product design analysis on virtual reality hardware platform with ENSIGHT, the 3D visualization software. At the same time, students may understand the advanced digital design method of high degree simulation in virtual experiment environment. All of these greatly improve the students' learning interest and enthusiasm, and cultivate students' engineering design ability and innovative thinking.

Virtual prototyping technology is the integration and combination of innovative design environment for running virtual simulation, including various simulation tools, analysis tools, control tools, methods for the cooperative work of equipment and organization. All these can improve products from concept design to dynamic simulation, even to the stage of the whole structure optimization. We can take advantage of virtual prototyping technology to build parts of the model, virtual assembly, and check the assembly clearance and interference. We can also use the analysis software to simulate the movement of the prototype and check the key parts. So we can find out errors in time, optimize the structure, and change the model conveniently. Thus, the virtual prototyping technology can improve the design efficiency, optimize design scheme, shorten the design cycle, and reduce the production cost. 
In the teaching process, we advocate the thought that the demand of mechanical innovation design drives virtual prototyping technology learning. And in accordance with the specific requirements to basic courses and professional courses, we emphasize the comprehensive training in students' ability in virtual modeling, motion simulation and optimization design. At the same time, combining with student curriculum design, graduate design and experimental skills project, participation in teacher's research projects, discipline competitions and other extracurricular practice, we organize the independent practice in the idea of progressive thinking and knowledge construction, to inspire students to autonomously study the corresponding tasks of product digital design in different stages, which can effectively solve the key problems that the undergraduates in mechanical majors are week in the practical ability of innovative design.

\section{Typical Virtual Experiment Analysis Of Mechanical Design}

Virtual assembly simulation experiment of typical parts. Products are made of several parts and components. In accordance with the provisions of the technical requirements, the labor process of synthesizing components by several parts or several parts and components to make the products, known as assembly. The former is called partial assembly and the latter is called general assembly. It generally includes assembly, adjustment, inspection and testing, painting, packaging, etc.

Assembly technological procedure is the processing file which regulates the planning and operation method of products or parts assembly, and it is the important basis for formulating the assembly plan and technical preparation, guiding specific work and dealing with issues. It has a positive effect on the quality of the assembly, the improvement of the production efficiency, the reduction of the cost and the labor intensity to the workers.

It includes analyzing original data on the assembly line, organizing assembly method, dividing assembly unit, obtaining the best assemble sequence, dividing assembly process, finishing assembly process documentation, developing product testing and testing standards.

The purpose of this experiment is to make the students understand the structure and relations of the typical part, know about assembly and virtual assembly, master the knowledge of assembly process, such as assembly sequence, restriction and interference, and skillfully use the mouse, keyboard, data glove and other interactive tools to grab, move, assemble, release and other operations to achieve product assembly and disassembly and verify the assembly process. It is shown in Fig.1and Fig.2.

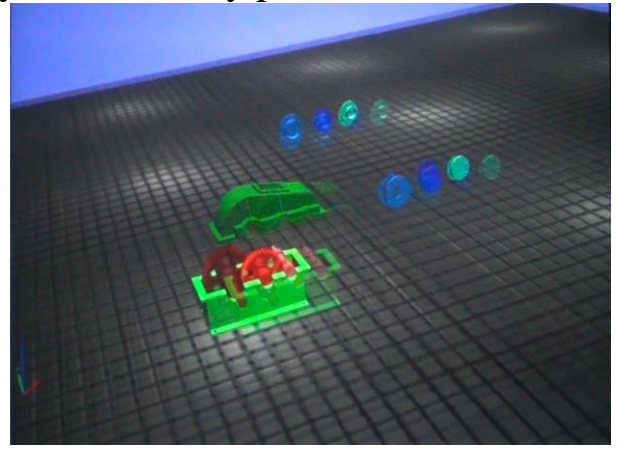

Figure 1. Virtual assembly simulation model of reduction box 


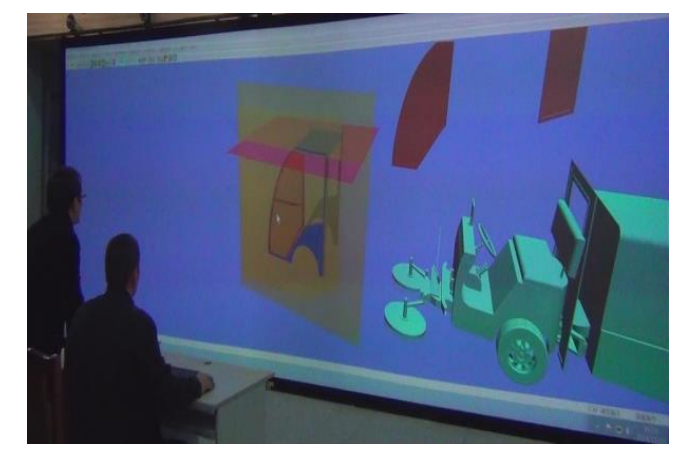

Figure 2. Virtual assembly of sweeping machine

Simulation experiment of the performance of overcoming obstacles for small multifunction landscape hedge trimer. The experiment is mainly used to research the process of vehicle overcoming obstacles. Through the interactive operation, it allows students to understand clearly the process of vehicle overcoming obstacles. This could help them learn the modeling and analysis method of ADAMS (Automatic Dynamic Analysis of Mechanical Systems), and preliminarily master the operation of adding motion constraint, and drive, simulation calculation and parameter measurement. At the same time, it will help students deepen the understanding of the corresponding knowledge, and master the method of post-processing and visualization of ENSIGHT. The meaning and function of virtual prototyping technology on product design is known by students.

Through the establishment of ADAMS motion model for the mini-hedge trimer, the simulation of overcoming obstacles and the post-processing and visualization of ENSIGHT, we can analyze intuitively the process of overcoming obstacles for small landscape hedge trimer. This experiment helps students to understand the method of the establishment and simulation for the motion model. First of all, students should import the existing 3D model to ADAMS. Second, define the material properties and constraints of each component. Then, set up the tire model and the ground file. The last, add the driver and set parameters to complete the simulation of the vehicle motion model. It is shown in Fig.3. Importing simulation data into ENSIGHT for post-processing and visualization by ADAMS to ENSIGHT interface generate more vivid animation in order to analyze on the virtual experimental platform.

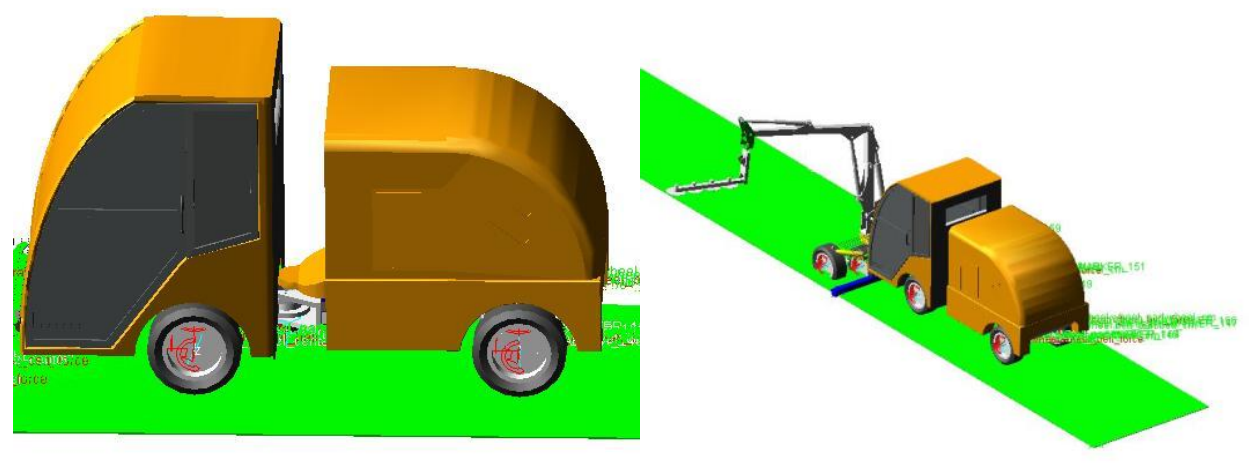

Figure 3. the model of simulation analysis is established 


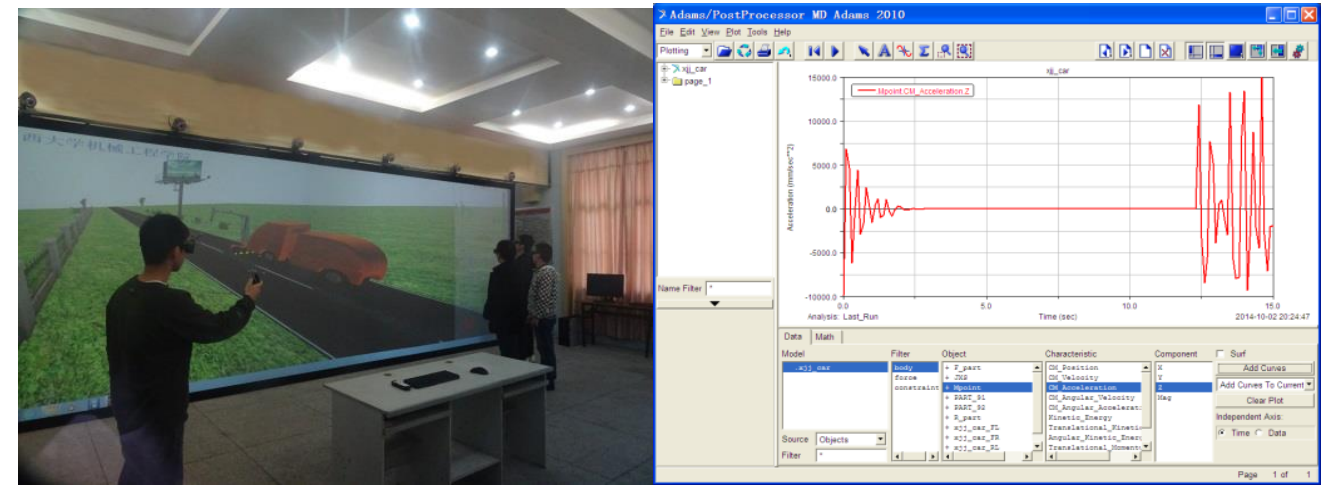

Fig. 4 simulation experiment of human computer interaction

In the experiment, the students could find the influence of velocity on the obstacle by changing the distance between obstacles and small pruning machine to simulate the process of the vehicle quickly overcoming obstacles. Students may also simulate the process of the vehicle overcoming obstacles, read and analyze the vertical acceleration of the small landscape hedge trimer by changing the size and height of obstacles. It is shown in Fig.4. Students can even further develop other motion simulation. Combining with the practical aspects of student extra-curricular, including curriculum design, graduation design, experimental skills project, students can be promoted to autonomously study the corresponding learning tasks, to realize the teaching of the machine design foundation and digital product innovation design based on virtual prototyping.

\section{Conclusions}

Experimental teaching research of virtual simulation of mechanical design emphasizes the idea of learning virtual prototyping technology which is dominated by the need of innovation design for machinery in the process of teaching, and pays attention to curricular learning. At the same time, combining with the practical aspects of student extra-curricular, including curriculum design, graduation design, experimental skills project, participation in teacher's research projects and discipline competitions, it organizes independent practice with the idea of progressive thinking and knowledge construction to promote students to autonomously learn the corresponding learning tasks of product digital design in different stage, and it effectively solve the key problems that the undergraduates in mechanical majors are week in the practical ability of innovative design.

\section{Acknowledgements}

This work is supported by the National Mechanical Engineering Virtual Simulation Experimental Teaching Center of Guangxi University and Guangxi New Century Higher Education Teaching Reform Project.

\section{References}

[1] P. Li, C.J. Mao, J. Xu, Construction of the national virtual simulation experiment teaching centers, improving the experimental teaching informatization in higher education, Research and Exploration in Laboratory. 32 (2013) 5-8. 
[2] Y.M. Meng, G.N. Qin, F.N. Lu, et.al, Designing the new experimental teaching system and establishing a mechanical engineering experimental teaching demonstration center, Research and Exploration in Laboratory, 1(2009)23-26.

[3] W.G. Wang, Construction consideration and suggestion of virtual simulation experimental teaching center, Research and Exploration in Laboratory, 32 (2013) 5-8.

[4] W.G. Cai, Application of virtual simulation technology in mechanical engineering experimental teaching, Experimental Technology and Management, 28(2011)

76-82.

[5] J.M. Zhai, X. Xu, P. Huang, et.al, Design and construction of remote experimental platform on mechanical basis, Experimental Technology and Management,29 (2012)84-89.

[6] Z.X. Han, L.B. Wei, H.B. Han, et.al, Study and practice on imitative and virtual experiment teaching, Experimental Technology and Management,23(2006) 64-66. 